\title{
TRPV4 contributes to ER stress and inflammation: implications for Parkinson's disease
}

\author{
Na Liu ${ }^{1,2}$, Liping Bai ${ }^{1}$, Zhipeng Lu' ${ }^{1}$, Rou Gu${ }^{1}$, Dongdong Zhao ${ }^{1}$, Fang Yan ${ }^{1}$ and Jie Bai ${ }^{1 *}$ (1)
}

\begin{abstract}
Background: Parkinson's disease (PD) is a progressive neurodegenerative disorder. Its molecular mechanism is still unclear, and pharmacological treatments are unsatisfactory. Transient receptor potential vanilloid 4 (TRPV4) is a nonselective $\mathrm{Ca}^{2+}$ channel. It has recently emerged as a critical risk factor in the pathophysiology of neuronal injuries and cerebral diseases. Our previous study reported that TRPV4 contributed to endoplasmic reticulum (ER) stress in the $\mathrm{MPP}^{+}$-induced cell model of PD. In the present study, we detected the role and the mechanism of TRPV4 in 1-Methyl4-phenyl-1,2,3,6-tetrahydropyridine (MPTP)-induced PD mice.

Methods: Intracerebral injection of an adeno-associated virus (AAV) into the substantia nigra (SN) of mice was used to knockdown or upregulate the expression of TRPV4 and intraperitoneal injection of MPTP. Rotarod and pole tests were used to evaluate the locomotor ability of mice. We used immunohistochemistry, Nissl staining and Western blot to detect the alterations in the number of tyrosine hydroxylase (TH)-positive neurons, Nissl-positive neurons, the levels of ER stress-associated molecules and proinflammatory cytokines in the SN.
\end{abstract}

Results: The SN was transfected with AAV for 3 weeks and expressed the target protein with green fluorescence. Knockdown of TRPV4 via injection of a constructed AAV-TRPV4 shRNAi into the SN alleviated the movement deficits of PD mice. Upregulation of TRPV4 via injection of a constructed AAV-TRPV4 aggravated the above movement disorders. The expression of TRPV4 was upregulated in the SN of MPTP-treated mice. Injection of AAV-TRPV4 shRNAi into the SN rescued the number of TH-positive and Nissl-positive neurons in the SN decreased by MPTP, while injection of AAVTRPV4 induced the opposite effect. Moreover, MPTP-decreased Sarco/endoplasmic reticulum Ca ${ }^{2+}$-ATPase 2 (SERCA2) and pro-cysteinyl aspartate specific proteinase-12 (procaspase-12), MPTP-increased Glucose-regulated protein 78 (GRP78), Glucose-regulated protein 94 (GRP94) and C/EBP homologous protein (CHOP) were inhibited by AAV-TRPV4 shRNAi infection, and enhanced by AAV-TRPV4. In the same way, MPTP-decreased procaspase-1, MPTP-increased Interleukin-18 (IL-18), Cyclooxgenase-2 (COX-2) and 5-Lipoxygenase (5-LOX) were inhibited by AAV-TRPV4 shRNAi, or further exacerbated by AAV-TRPV4.

Conclusions: These results suggest that TRPV4 mediates ER stress and inflammation pathways, contributing to the loss of dopamine (DA) neurons in the SN and movement deficits in PD mice. Moreover, this study provides a new perspective on molecular targets and gene therapies for the treatment of PD in the future.

Keywords: TRPV4, MPTP, Parkinson's disease, SN, ER stress, Inflammation

*Correspondence: jiebai662001@126.com

1 Medical School, Kunming University of Science and Technology, No.727

Jingming South Road, Kunming 650500, China

Full list of author information is available at the end of the article permits use, sharing, adaptation, distribution and reproduction in any medium or format, as long as you give appropriate credit to the original author(s) and the source, provide a link to the Creative Commons licence, and indicate if changes were made. The images or other third party material in this article are included in the article's Creative Commons licence, unless indicated otherwise in a credit line to the material. If material is not included in the article's Creative Commons licence and your intended use is not permitted by statutory regulation or exceeds the permitted use, you will need to obtain permission directly from the copyright holder. To view a copy of this licence, visit http://creativecommons.org/licenses/by/4.0/. The Creative Commons Public Domain Dedication waiver (http://creativeco mmons.org/publicdomain/zero/1.0/) applies to the data made available in this article, unless otherwise stated in a credit line to the data. 


\section{Background}

Parkinson's disease (PD) is a common neurodegenerative disease characterized by the loss of dopamine (DA) neurons in the substantia nigra (SN) and a decrease in DA content in the striatum. The pathogenesis of PD is related to environmental factors. 1-Methyl-4-phenyl-1,2,3,6-tetrahydropyridine (MPTP), an environmental neurotoxin, is widely used to produce the animal models for the study of PD, especially the rodent models $[1,2]$. Although the mechanism of PD remains elusive, increasing evidence indicates that ER stress is involved [3, 4]. The endoplasmic reticulum (ER) plays a crucial role in maintaining cellular function and $\mathrm{Ca}^{2+}$ homeostasis. When ER stress occurs, the $\mathrm{Ca}^{2+}$-induced excitotoxicity or $\mathrm{Ca}^{2+}$ overload is considered as an essential mechanism linked to neuronal apoptosis. Our previous study showed that upregulation of TRPV4 induced by the 1-methyl-4-phenylpyridinium ion $\left(\mathrm{MPP}^{+}\right)$mediated ER stress and contributed to PC12 cell apoptosis [5]. The role of TRPV4 in regulating neurotoxicity in vitro was first reported in the study. It was also identified that HC067047 (a special inhibitor of TRPV4) had an antiapoptotic effect. This supports the view that disorder of $\mathrm{Ca}^{2+}$ homeostasis is a primary feature of the pathogenesis of PD but not the result of the neurodegenerative process. In addition, neuroinflammation refers to the inflammatory process occurring in nervous system. Clinical data indicate higher levels of the proinflammatory cytokines interleukin-6 (IL-6) and interleukin-1 $\beta$ (IL-1 $\beta$ ) and tumor necrosis factor- $\alpha$ (TNF- $\alpha)$ in the serum and cerebrospinal fluid of PD patients $[6,7]$. Neuroinflammation has been linked to PD and is responsible for DA neuron death in the SN. In addition to astrocytes and microglia, neurons also release cytokines and chemokines, and induce a robust inflammatory response [8]. Correspondingly, one study has shown that the dysfunction of $\mathrm{Ca}^{2+}$ homeostasis is responsible for the high levels of inflammatory factors leading to epileptic activity [9]. Moreover, inhibiting transient receptor potential (TRP) channel may decrease the influx of $\mathrm{Ca}^{2+}$ and regulate morphine-induced neurodegeneration of neurons in the hippocampus by decreasing inflammation [10].

Transient receptor potential vanilloid 4 (TRPV4), a member of the TRP family, is a nonselective $\mathrm{Ca}^{2+}$ channel involved in diverse pathological and physiological responses. It is more permeable to $\mathrm{Ca}^{2+}$ than other cations [11]. It is widely expressed in neurons in the brain $[12,13]$. When it is activated by endogenous and exogenous stimuli, the influx of $\mathrm{Ca}^{2+}$ is increased and results in overloaded of intracellular free $\mathrm{Ca}^{2+}[14,15]$. Therefore, hyperactivation of TRPV4 leads to cell apoptosis in many pathological processes [16-18] and acts as a risk factor for the development of brain diseases [19], including cerebral ischemic reperfusion injury [20,21], intracerebral hemorrhage [22], brain edema [23, 24], epilepsy [25], and Alzheimer's disease [26, 27]. In contrast, the TRPV4 antagonist $\mathrm{HC} 067047$ exerts a neuroprotective effect by inhibiting the unfolded protein response (UPR) and the inflammation signaling pathways [22, 25]. Thus, the contribution of TRPV4 on cytotoxicity has received more attention from researchers. However, to the best of our knowledge, the role of TRPV4 in regulating neurotoxicity in the MPTP-induced mouse model is unknown.

Neurodegenerative diseases such as PD impose a heavy burden on the aging society. The currently available treatment approaches for PD are limited. PD is normally controlled by drugs. However, as PD continues to advance, presently available pharmacotherapies often fail to effectively relieve symptoms and sometimes cause side effects $[28,29]$. The new treatment strategies for PD are urgently needed.

Based on a previous study in vitro, we stereotaxically injected AAV2/9 bilaterally into the SN of mice to regulate TRPV4 gene expression and then intraperitoneally injected MPTP. We examined the expression of TRPV4 and assessed its contribution to ER stress and inflammation induced by MPTP in vivo. These results improve the understanding on the pathogenesis of $\mathrm{PD}$ and provide a novel therapeutic target with PD.

\section{Methods \\ Animals}

Male C57BL/6J mice (20-25 g, 7-8 weeks) were obtained from Chongqing Medical University (Chongqing, China). Mice were housed individually in a plastic cage under a $12 \mathrm{~h}$ light/dark cycle (lights on at 8:00 a.m.) with ad libitum access to water and food. The housing room temperature was maintained at $20-26^{\circ} \mathrm{C}$, and the humidity was $40-70 \%$. The animals were acclimatized to this laboratory environment for at least 1 week prior to experimental procedures. The experimenter was blind to the treatment groups at the time of the tests and analyses. Surgeries and behavioral assays were carried out at the Laboratory Animal Center Facilities of Kunming University of Science and Technology.

\section{Drug treatment and viral vectors}

MPTP was purchased from Sigma-Aldrich (\# M0896). AAV2/9 TRPV4 shRNA $\left(1.5 \times 10^{12} \mathrm{vg} / \mathrm{ml}\right.$, AAV-TRPV4 shRNAi) was constructed to knockdown TRPV4, and AAV2/9 TRPV4 $\left(1.6 \times 10^{12} \mathrm{vg} / \mathrm{ml}\right.$, AAV-TRPV4) was used to upregulate TRPV4 expression, and were purchased from Hanbio Biotechnology Co. (Shanghai, China). 


\section{Animal model}

For AAV2/9 injection, mice were anesthetized with pentobarbital sodium salt $(50 \mathrm{mg} / \mathrm{kg})$ through intraperitoneal injections and placed in a stereotaxic frame (RWD Life Science Co, Ltd, China). The mice were infused with $0.5 \mu \mathrm{l}$ AAV-TRPV4 shRNAi or $0.3 \mu \mathrm{l}$ AAV-TRPV4 per side into the $\mathrm{SN}$ (3.0 mm posterior to bregma; $1.0 \mathrm{~mm}$ lateral to the midline; $4.5 \mathrm{~mm}$ below the dural surface) [30] with using an electric microinjection pump (World Precision Instruments Inc. Shanghai, China) at a rate of $250 \mathrm{nl} / \mathrm{min}$, and the needle was left in place for an additional $5 \mathrm{~min}$ period before it was slowly retracted. After 21 days, we randomly selected one mouse from each AAV-injected group to obtain frozen sections, and confirmed the accuracy of transfection site by green fluorescent protein (GFP) under a fluorescence microscope (Leica, Germany). Based on the previous study, the left mice were administered with MPTP (30 mg/kg) and saline by intraperitoneal injections for 7 days at an interval of $24 \mathrm{~h}$ [31].

\section{Fluorescence imaging}

The mice were sacrificed by cervical vertebra dislocation. To obtain frozen sections, the brains were fixed in $4 \%$ paraformaldehyde (PFA) solution overnight at $4{ }^{\circ} \mathrm{C}$ and subsequently incubated in $30 \%$ sucrose to dehydrate overnight at $4{ }^{\circ} \mathrm{C}$. Then, the brains were frozen in the freezing microtome (Leica, Germany), and the samples were cut into coronal slices at $25 \mu \mathrm{m}$ thickness in the region $-3.00 \mathrm{~mm}$ from the bregma for examination GFP under a fluorescence microscope (Leica, Germany).

\section{Movement behavioral tests \\ Pole test}

A vertical wooden pole with a rough surface $(5 \mathrm{~cm}$ in height and $1 \mathrm{~cm}$ in diameter) was placed in the home cage. The mice were placed head-up on top of the pole, oriented themselves downward and descended the length of the pole back into their home cages. The mice received 2 days of training consisting of three trials for each session day. On the test day, the mice were subjected to three trials, and the time spent orienting themselves downward ( $T$-turn time) and the time spent descending ( $T$-descend time) were measured. The best performance over the three trials was used. If the mouse was unable to turn completely downwards, fall or slip down, the default value of $120 \mathrm{~s}$ was recorded, which represented the maximal severity of impairment [32, 33].

\section{Rotarod test}

Motor performance was tested using an accelerating rotarod (Ugo Basile, Italy). Prior to the test, the mice were trained for 2 days. On the training day, the mice were placed on the rod rotating at a speed of 5-25 rpm for three adaptation sessions, and the duration did not exceed $180 \mathrm{~s}$. At least $5 \mathrm{~min}$ of rest was allowed between each trial to alleviate mouse fatigue and stress. On the test day, the mice were subjected to three trials in which the rod accelerated $(5-30 \mathrm{rpm})$. The mice were placed on the rod, and the time it took each mouse to fall from the rod was recorded. The average time was calculated for each mouse and then used for the statistical purpose.

\section{Immunohistochemistry}

The mice were anesthetized with pentobarbital sodium salt $(50 \mathrm{mg} / \mathrm{kg})$ by intraperitoneal injections and slowly perfused through the left ventricle with $0.9 \%$ saline at room temperature, followed by ice-cold 4\% PFA solution. The brains were rapidly removed and fixed in $4 \%$ PFA solution overnight at $4{ }^{\circ} \mathrm{C}$. After fixation, brains were embedded in paraffin following standard procedures and serially sectioned at $6 \mu \mathrm{m}$ (Leica, Germany) for staining. Coronal sections corresponding to the region -2.96 to $-3.06 \mathrm{~mm}$ from the bregma were selected for analysis. Every 7th and 9th section was used for immunohistochemistry and Nissl staining, respectively. In immunohistochemistry assay, brain sections were deparaffinized with xylene and rehydrated through a serious of ethanol solutions (from 100 to $75 \%$ ). Typically, the slices were immersed in xylene for $12 \mathrm{~min}$ (twice), 100\% ethanol for $5 \mathrm{~min}$, 95\% ethanol for $5 \mathrm{~min}, 85 \%$ ethanol for $5 \mathrm{~min}$, and $70 \%$ ethanol for $5 \mathrm{~min}$ and then washed with ultrapure water for $5 \mathrm{~min}$ three times. Antigen retrieval was performed by soaking the sections in boiling citrate buffer ( $\mathrm{pH}$ 6.0) in a microwave for $15 \mathrm{~min}$. Endogenous peroxidase activity was blocked with $3 \%$ hydrogen peroxide $\left(\mathrm{H}_{2} \mathrm{O}_{2}\right)$ for $30 \mathrm{~min}$. The sections were permeabilized with $0.25 \%$ Triton-X-100 in phosphate-buffered saline (PBS) for $10 \mathrm{~min}$, followed by incubation with $5 \%$ normal goat serum (Thermo Fisher, USA) for $1 \mathrm{~h}$ at room temperature. Next, the brain sections were probed with antiTyrosine hydroxylase (TH) (1:300; ab137869, Abcam) primary antibody solution at $4{ }^{\circ} \mathrm{C}$ overnight. Then, the sections were incubated with secondary antibody (goat anti-rabbit IgG, 1:200; \#5450-0010, KPL, USA) at room temperature for $1 \mathrm{~h}$. After being incubated with 3,3'-diaminobenzidine (DAB) (Thermo Fisher, USA) for $10 \mathrm{~min}$, the sections were counterstained with hematoxylin. Typically, the sections were rinsed three times in PBS for $5 \mathrm{~min}$ between each incubation period. Images were taken with using a Leica DM1000 microscope (Leica, China). 


\section{Nissl staining}

The slices were dewaxed according to the method mentioned above. Next, the slices were immersed in $2 \%$ thionine for $3 \mathrm{~min}$, ultrapure water for $10 \mathrm{~s}$ (twice), 100\% ethanol for $10 \mathrm{~s}, 95 \%$ ethanol for $10 \mathrm{~s}, 85 \%$ ethanol for $10 \mathrm{~s}, 70 \%$ ethanol for $10 \mathrm{~s}$, xylene for $2 \mathrm{~min}$, and $100 \%$ ethanol for $10 \mathrm{~s}$ and then washed with ultrapure water for $5 \mathrm{~min}$. Finally, the slices were sealed with neutral gum after desiccation.

\section{Stereology and neuron counting}

An unbiased stereological estimation of the total number of TH-positive neurons and Nissl-positive neurons in the $\mathrm{SN}$ was performed using the optical fractionator method and calculated with Stereo Investigator software (MBF Bioscience). Counting was performed using microscope (10 $\times$ objective lens). The counted area covered from the rostral tip of substantia nigra pars compacta to the caudal end of substantia nigra pars reticulate. The counting frame was randomly placed on the first counting field and gradually moved through all counting areas. The total number of neurons was estimated according to the optical fractionator formula.

\section{Western blot analysis}

The mice were sacrificed by cervical vertebra dislocation after behavioral tests.

The SN was rapidly dissected out, frozen, and stored in a deep freezer at $-80^{\circ} \mathrm{C}$ until analysis. Samples were lysed in the fresh radio immunoprecipitation assay (RIPA) protein lysis buffer. The protein concentration was determined using Bio-Rad protein assay reagent (Hercules, CA, USA). Proteins were separated by sodium dodecyl sulfate-polyacrylamide gel electrophoresis (SDS-PAGE) and transferred onto polyvinylidene fluoride (PVDF) membranes (Millipore Corporation, Billerica, MA, USA). Then, the membranes were soaked in a solution of $10 \%$ skim milk or $3 \%$ bovine albumin $\mathrm{V}$ (in PBS, pH 7.2, containing 0.1\% Tween-20) overnight at $4{ }^{\circ} \mathrm{C}$. The membranes were incubated by primary antibodies against TRPV4 (1:1000; ab39260, Abcam), sarco/endoplasmic reticulum $\mathrm{Ca}^{2+}$-ATPase 2 (SERCA2, 1:2000; ab137020, Abcam), glucose-regulated protein 78 (GRP78, 1:1000; \#11587-1-AP, Proteintech Group), glucose-regulated protein 94 (GRP94, 1:1000; \#10979-1-AP, Proteintech Group), pro-cysteinyl aspartate specific proteinase-12 (procaspase-12, 1:1000; ab8118, Abcam), C/ EBP homologous protein (CHOP, 1:1000; \#15204-1-AP, Proteintech Group), TH (1:1500; ab137869, Abcam), procysteinyl aspartate specific proteinase-1 (procaspase-1, 1:1500; ab238972, Abcam), Cyclooxgenase-2 (COX-2, 1:1000; \#66351-1-lg, Proteintech Group), 5-lipoxygenase (5-LOX, 1:1000; ab169755, Abcam), Interleukin-18
(IL-18, 1:1000; ab207323, Abcam) and $\beta$-actin (1:10,000; ab115777, Abcam) overnight at $4{ }^{\circ} \mathrm{C}$. After extensive washing, the membranes were incubated with peroxidase conjugated goat anti-mouse or anti-rabbit IgG (\#5450-0011, \#5450-0010 KPL, USA). The epitopes were visualized with an enhanced chemiluminescence (ECL) Western blot detection kit (Millipore Corporation, USA). Other steps were performed following the instructions of each antibody. Densitometry analysis was performed using ImageJ software.

\section{Statistical analysis}

Statistical analyses were performed by Prism 5 (GraphPad Software). Before data analysis, both normality of the distribution and homogeneity of variance were assessed. All data are presented as mean \pm SEM. For assessment of change of a group by a certain intervention, data were analyzed by the two-tailed Student's $t$ test for comparison differences between the control group and the treated group. Data were analyzed by one-way variance (ANOVA) with Bonferroni post hoc test for comparison between multiple groups. $P$ value less than 0.05 was considered statistically significant. The significance level is represented as asterisks $\left({ }^{*} P<0.05,{ }^{* *} P<0.01,{ }^{* * *} P<0.001\right)$.

\section{Results}

\section{TRPV4 expression was upregulated in MPTP-induced PD}

mice

Based on our previous study in vitro, we first detected the expression of TRPV4 in the SN of MPTP-induced mice. We found that the level of TRPV4 was significantly increased compared with that in vehicle-treated control mice (Fig. 1A). This result suggests that TRPV4 may play an essential role in MPTP-induced neurotoxicity. To further confirm this hypothesis, we next infused AAVGFP-packaged mouse shRNAi or the full-length TRPV4 gene by intracerebral injection to knockdown or upregulate the expression of TRPV4 in the $\mathrm{SN}$, respectively. By 3 weeks post AAV injection, the SN was transfected and expressed the target protein with green fluorescence (Fig. 1B). Subsequently, we administered MPTP intraperitoneally following the schedule below (Fig. 1C).

\section{The effect of TRPV4 on the behavioral performance of PD mice}

The pole and rotarod tests are used to assess the agility and bradykinesia in PD mice and could sensitively detect nigrostriatal dysfunction [33]. The results of the tests indicated that behavioral performances were significantly different among the groups. Post hoc analysis revealed that motor disorders were induced in the group exposed to MPTP for 7 days compared with the control group. Knockdown of TRPV4 alleviated the 


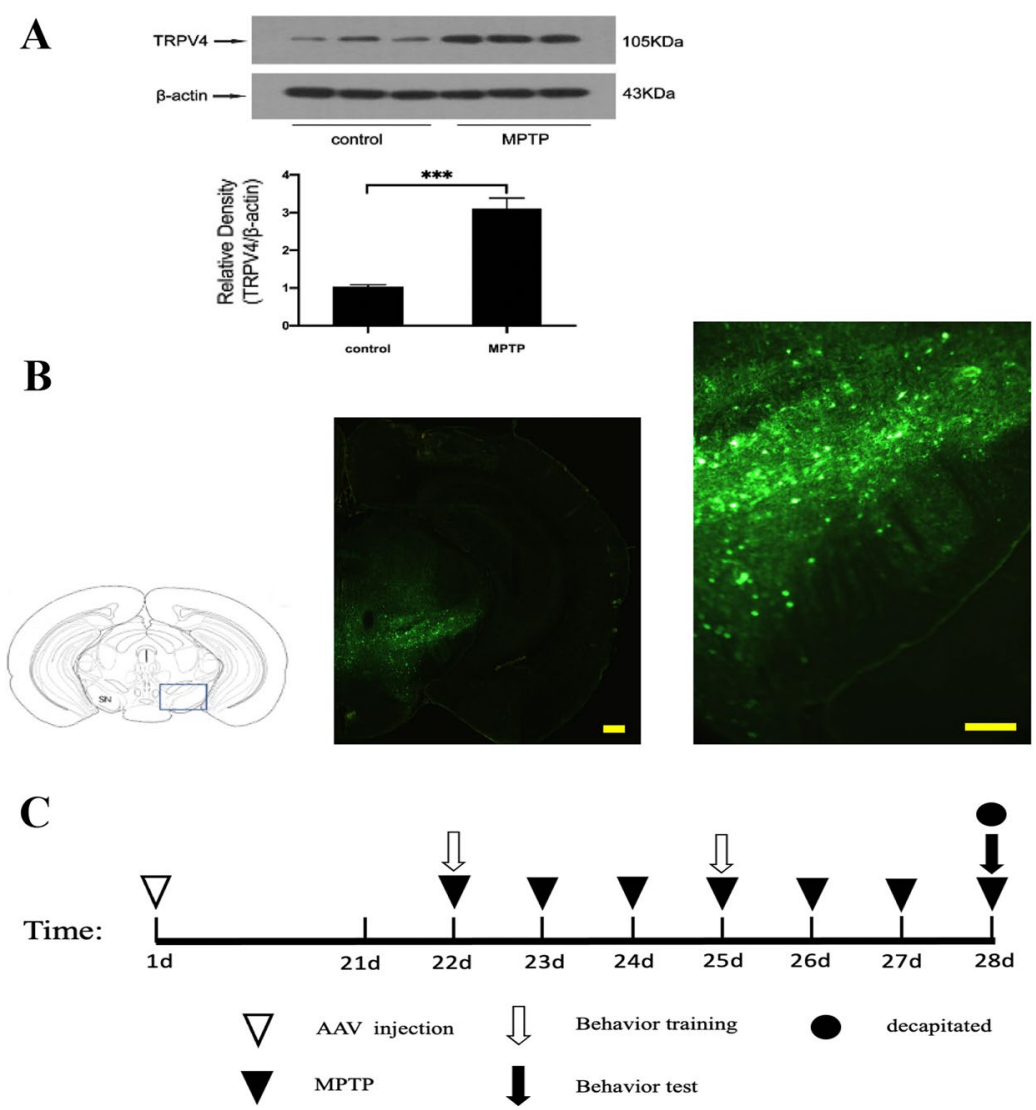

Fig. 1 Expression of TRPV4 in the SN after treatment with MPTP and AAV injection. A MPTP was administrated intraperitoneally at $30 \mathrm{mg} / \mathrm{kg} / \mathrm{day}$ for 7 days, mice were sacrificed after the behavioral tests by cervical vertebra dislocation, the SN was rapidly dissected out. The expression of TRPV4 in the SN was assessed by Western blot $(n=10)$. Compared to that in the control group, TRPV4 was upregulated by MPTP. Data are presented as mean \pm SEM. Statistical significance: ${ }^{* *} P<0.001$. B AAV $0.5 \mu \mathrm{l}$ was stereotaxically injected bilaterally into the SN at a rate of $250 \mathrm{nl} / \mathrm{min}, 21$ days later the brains were dissected and cut into coronal slices at $25 \mu \mathrm{m}$ thickness, fluorescence images of coronal frozen sections at different magnifications were presented by GFP in neurons in the SN region. Scale bar $=100 \mu \mathrm{m}$. C Schematic illustration of the experimental schedule

movement deficits of PD mice. AAV-TRPV4 shRNAi restored the $T$-tune time, the $T$-descend time and the retention time of PD mice (Fig. 2A, C, E). Upregulation of TRPV4 further prolonged the $T$-tune time and the $T$-descend time, and greatly shortened the retention time of PD mice (Fig. 2B, D, F). These motor alterations indicated a pivotal role of TRPV4 in movement deficits in PD mice.

\section{TRPV4 expression was significantly correlated} with TH-positive neuron survival in the SN

To explore the role of TRPV4 in PD mice, we regulated the expression of TRPV4 in the SN by AAV transfection. By Western blot, we found that TRPV4 expression was changed mainly in the $\mathrm{SN}$ in each group (Fig. 3A, B). Furthermore, knockdown of TRPV4 rescued the number of $\mathrm{TH}$-positive neurons in the $\mathrm{SN}$, while upregulation of TRPV4 exacerbated the reduction in the number of
TH-positive neurons in PD mice (Fig. 3C). These results were confirmed by detecting the expression of $\mathrm{TH}$ by Western blot (Fig. 3D, E).

\section{TRPV4 expression was significantly correlated} with neurodegeneration in the SN

To further determine the role of TRPV4 in PD mice, we performed Nissl staining in the SN. We found that knockdown of TRPV4 rescued the number of Nissl-positive neurons, while upregulation of TRPV4 exacerbated the decrease in the number of Nissl-positive neurons in the SN of PD mice (Fig. 4). Stereological analysis showed that TRPV4 played a crucial role in neurodegeneration.

\section{Knockdown of TRPV4 in the SN attenuated ER stress induced by MPTP}

Based on the findings presented above, we then investigated whether TRPV4 in the SN could serve as a key determinant of ER stress and thus induce the loss of 

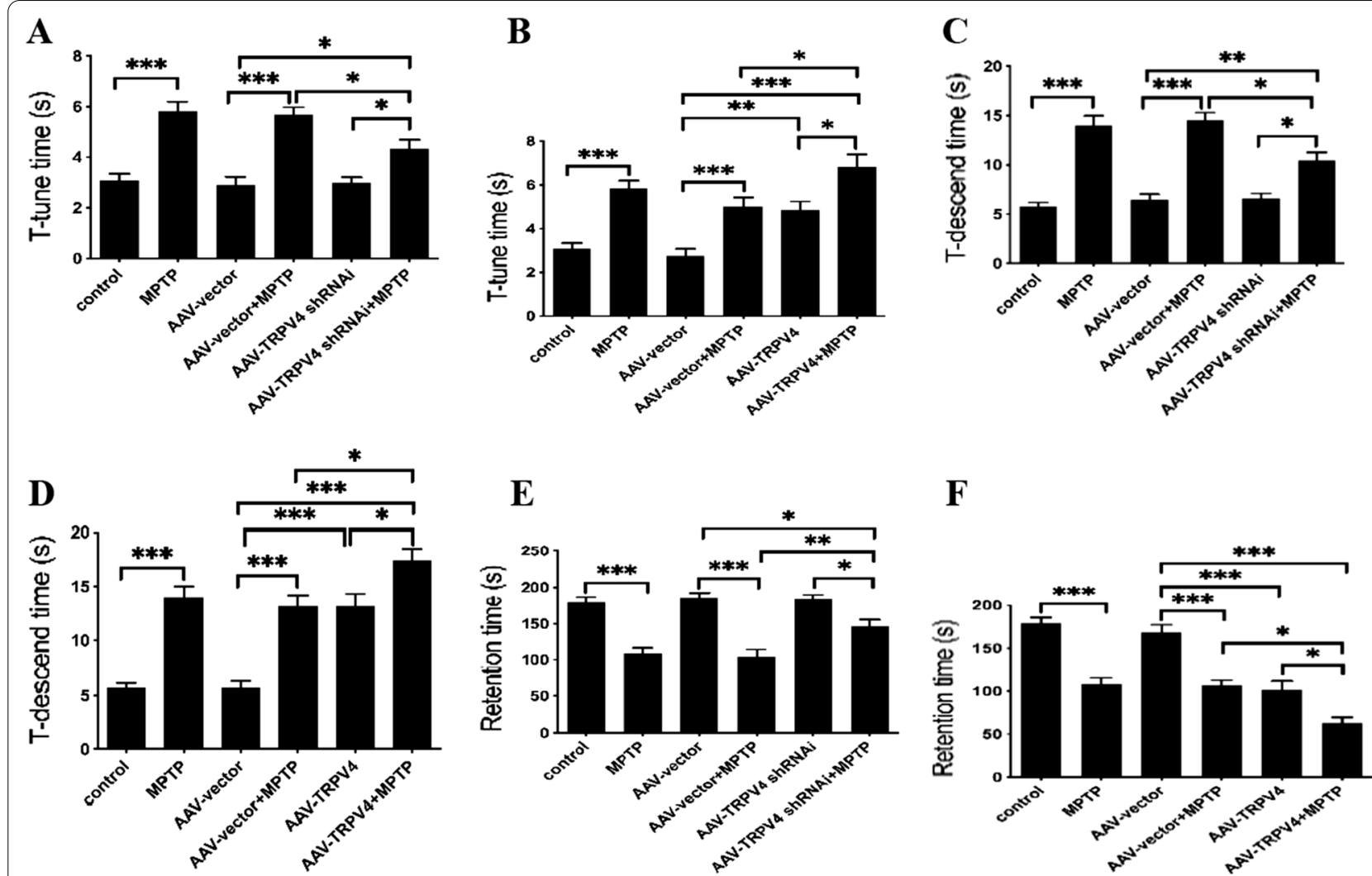

Fig. 2 TRPV4 affected the behavioral performances of PD mice. After infused with $0.5 \mu$ IAAV-TRPV4 shRNAi or $0.3 \mu$ IAAV-TRPV4 bilaterally into the SN for 21 days and administrated with MPTP at $30 \mathrm{mg} / \mathrm{kg} /$ day for 7 days, mice were subjected to movement behavioral tests, including the pole test and rotarod test. A Knockdown of TRPV4 restored the T-tune time of PD mice $(n=12)$. B Upregulation of TRPV4 further prolonged the T-tune time of PD mice $(n=12)$. C Knockdown of TRPV4 restored the T-descend time of PD mice $(n=12)$. D Upregulation of TRPV4 further prolonged the $T$-descend time of PD mice $(n=12)$. E Knockdown of TRPV4 restored the retention time of PD mice $(n=12)$. $\mathbf{F}$ Upregulation of TRPV4 further shortened the retention time of PD mice $(n=12)$. For each condition, data were collected from three trials for each mouse. All data are presented as mean \pm SEM. Significant differences: ${ }^{*} P<0.05,{ }^{* *} P<0.01,{ }^{* * *} P<0.001$

neurons. To examine this possibility, we constructed an AAV2/9 TRPV4 shRNAi, which was infused bilaterally into the $\mathrm{SN}$ for 21 days to knockdown TRPV4 expression. We found that the expression of SERCA2, which is located on the ER and associated with ER stress, was decreased by MPTP and restored by knockdown of TRPV4 (Fig. 5A). Moreover, MPTP-induced activation of the ER-resident chaperones GRP78 and GRP94 was inhibited by AAV-TRPV4 shRNAi infection (Fig. 5B, C), suggesting that knockdown of TRPV4 attenuated MPTPinduced ER stress. To further validate this mechanism of action of TRPV4, we assessed the expression of CHOP and procaspase-12, which are key apoptotic factors associated with ER stress. Knockdown of TRPV4 decreased

(See figure on next page.)

Fig. 3 TRPV4 affected the number of TH-positive neurons in the SN. After infused with $0.5 \mu$ AAV-TRPV4 shRNAi or $0.3 \mu$ I AAV-TRPV4 bilaterally into the SN of mice for 21 days and administrated with MPTP at $30 \mathrm{mg} / \mathrm{kg} /$ day for 7 days, the SN was rapidly dissected out. The expressions of TRPV4 and TH in the SN were detected by Western blot. A AAV-TRPV4 shRNAi downregulated the expression of TRPV4 in the SN, and significantly inhibited the MPTP-induced increase in TRPV4 $(n=6)$. B AAV-TRPV4 upregulated the expression of TRPV4 in the SN, and further increased the MPTP-induced high levels of TRPV4 $(n=6)$. C Representative sample of brain slices with immunohistochemistry staining of TH-positive neurons in the SN of mice. Knockdown of TRPV4 rescued the number of TH-positive neurons, while upregulation of TRPV4 exacerbated the already reduction in the number of TH-positive neurons in the SN of PD mice $(n=3)$. D Knockdown of TRPV4 restored the expression of TH in the SN of PD mice $(n=6)$. E Upregulation of TRPV4 further decreased the expression of TH in the SN of PD mice $(n=6)$. All data are presented as mean \pm SEM. Significant differences: ${ }^{*} P<0.05$, ${ }^{*} P<0.01,{ }^{* * *} P<0.001$ 

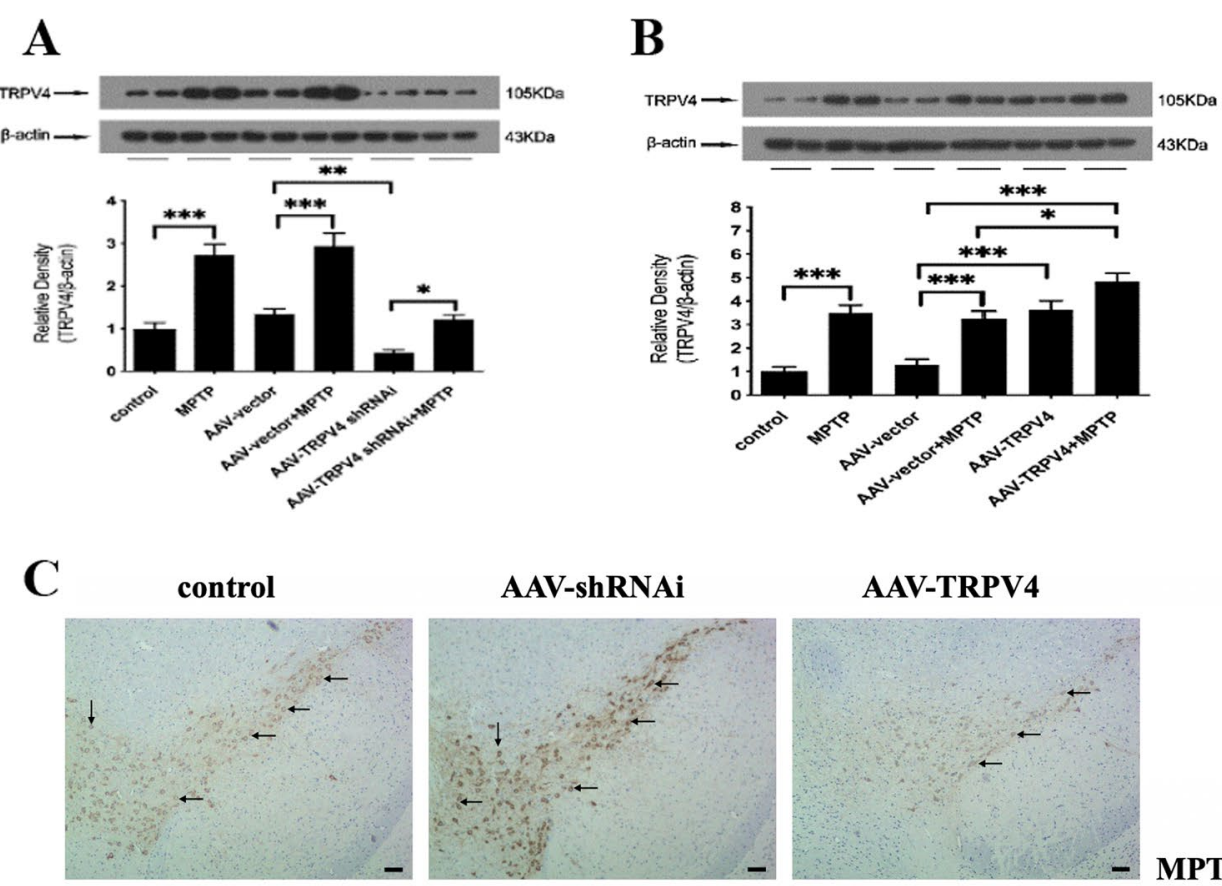

AAV-shRNAi

AAV-TRPV4
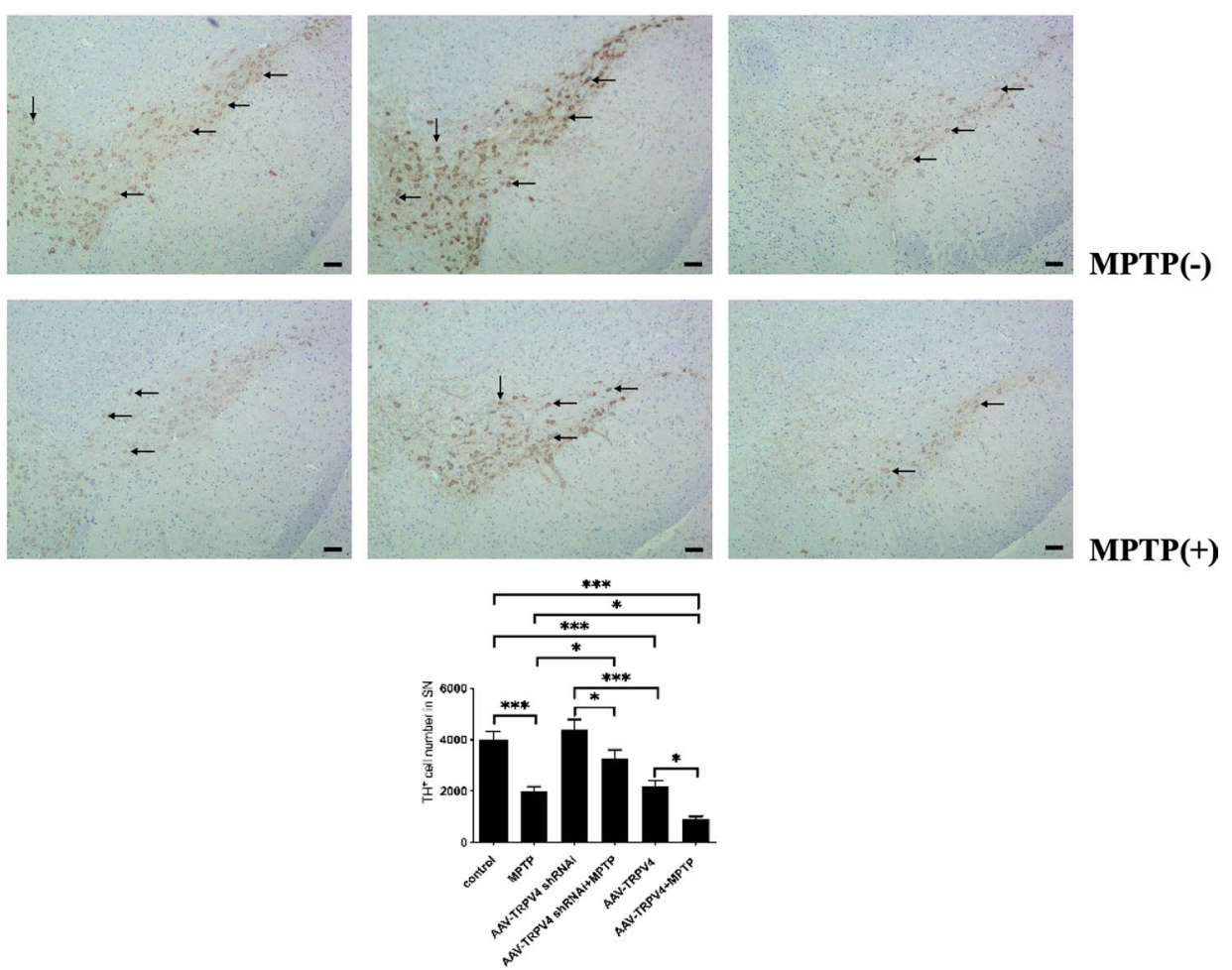

D

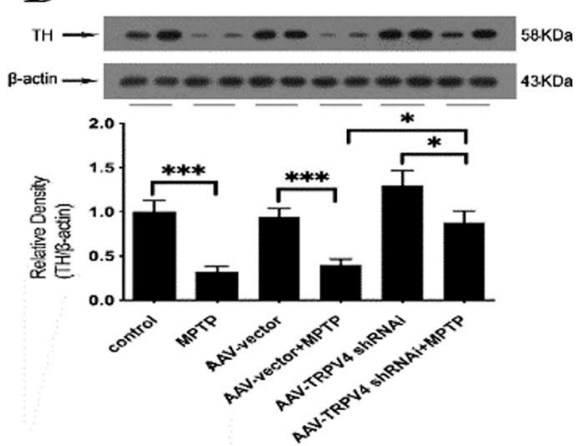

E

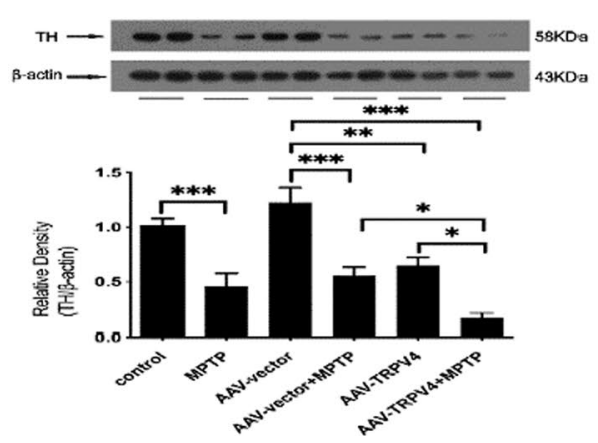

Fig. 3 (See legend on previous page.) 

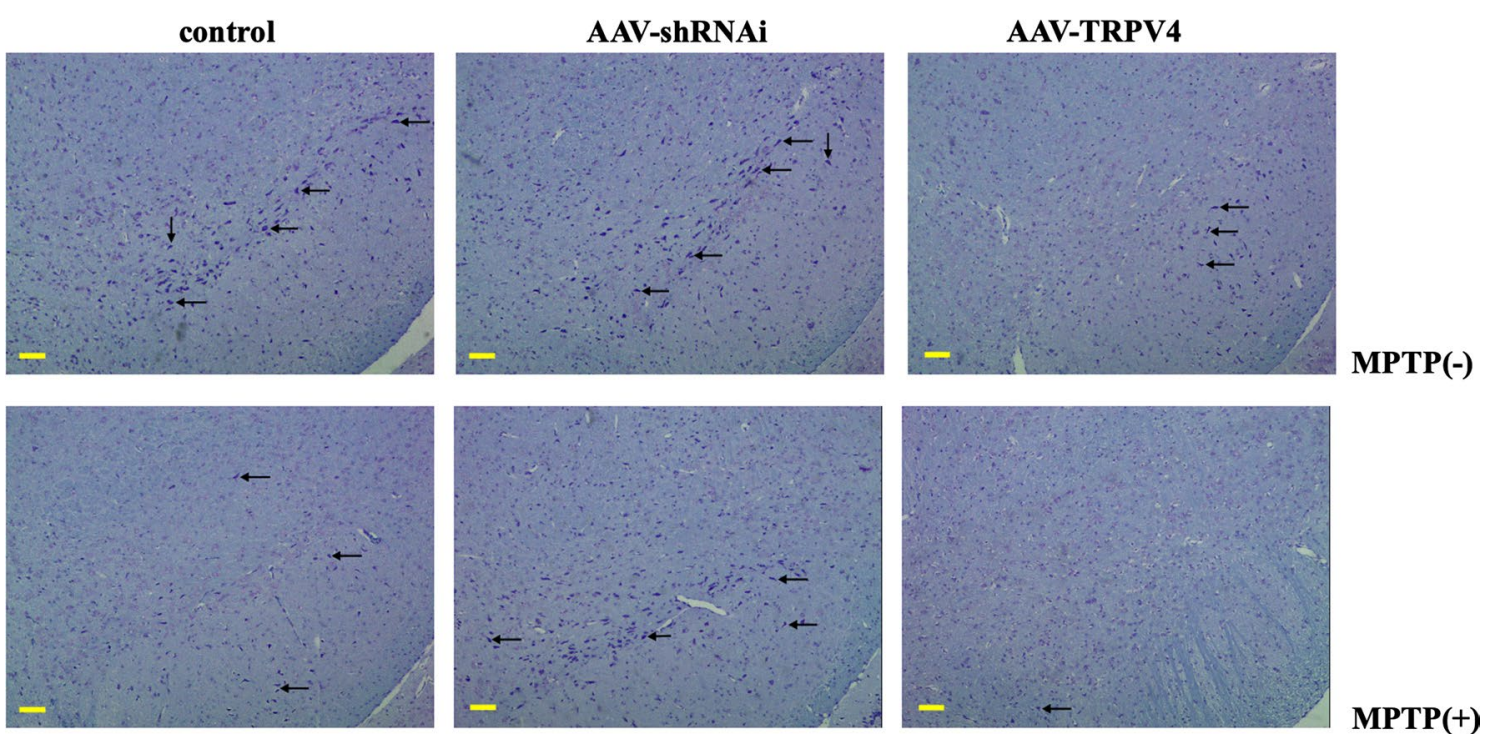

$\operatorname{MPTP}(+)$

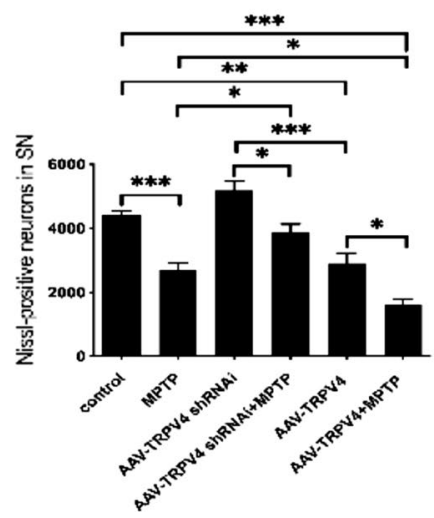

Fig. 4 TRPV4 affected the number of Nissl-positive neurons in the SN. After infused with $0.5 \mu$ I AAV-TRPV4 shRNAi or $0.3 \mu$ I AAV-TRPV4 bilaterally into the SN of mice for 21 days and administrated with MPTP at $30 \mathrm{mg} / \mathrm{kg} /$ day for 7 days, representative sample of brain slices with Nissl staining showed Nissl-positive neurons in the SN. Knockdown of TRPV4 rescued the number of Nissl-positive neurons in the SN of PD mice, while upregulation of TRPV4 exacerbated the already reduction in the number of Nissl-positive neurons in the SN of PD mice (bar: $100 \mu \mathrm{m}, n=3)$. Data are presented as mean \pm SEM. Significant differences: ${ }^{*} P<0.05,{ }^{* *} P<0.01,{ }^{* * *} P<0.001$

the expression of $\mathrm{CHOP}$ and increased procaspase-12 expression in PD mice (Fig. 5D, E). Taken together, these data indicate that knockdown of TRPV4 exerts its neuroprotective effect by attenuating MPTP-induced ER stress.

\section{Upregulation of TRPV4 in the SN further aggravated ER stress induced by MPTP}

To further confirm that TRPV4 has a direct effect on ER stress independent of MPTP-induced neurotoxicity, we injected AAV2/9 TRPV4 to upregulate the expression of TRPV4 in the SN. Upregulation of TRPV4 also induced ER stress. Activation of TRPV4 significantly decreased SERCA2 (Fig. 6A), activated GRP78, GRP94, CHOP and procaspase-12 (Fig. 6B-E). Moreover, we found that upregulation of TRPV4 in PD mice accelerated these changes. These data indicate that TRPV4 is an independent risk factor affecting ER homeostasis and that its effect does not rely on MPTPinduced neurotoxicity.

\section{Knockdown of TRPV4 in the SN attenuated inflammation induced by MPTP}

To determine whether TRPV4 plays an important role in MPTP-induced inflammation in the $\mathrm{SN}$, we detected the levels of proinflammatory factors by Western blot. We found that knockdown of TRPV4 before MPTP treatment inhibited the MPTP-induced activation of procaspase-1 (Fig. 7A) and significantly reduced the high protein levels of IL-18, COX-2, and 5-LOX in the $\mathrm{SN}$ of PD mice (Fig. 7B-D). These results indicate that 


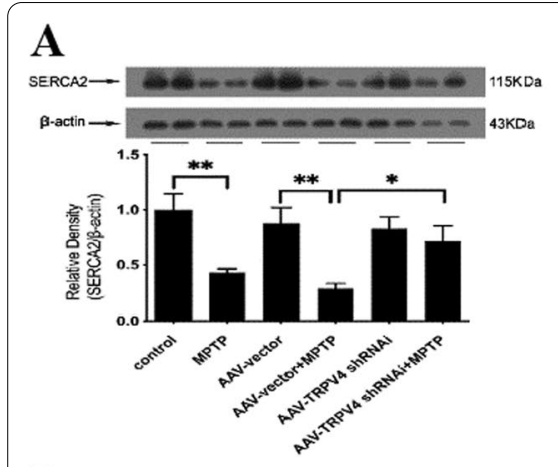

D

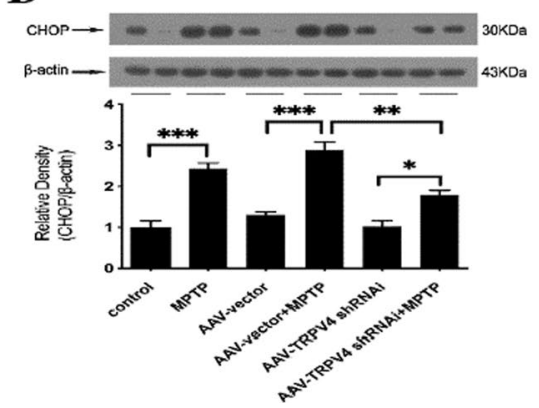

B

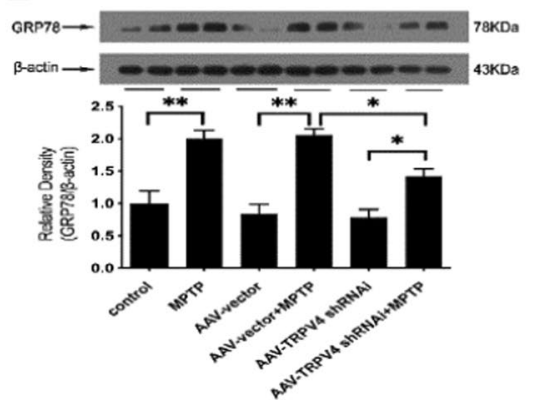

E

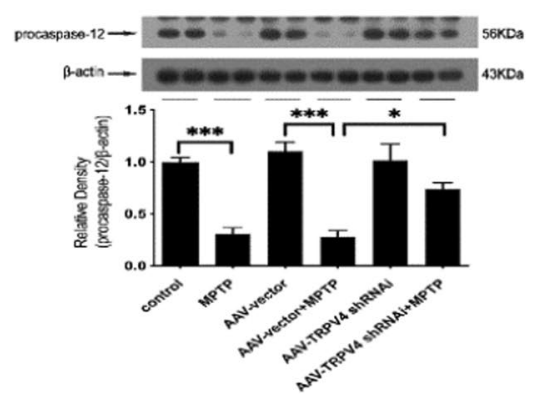

C

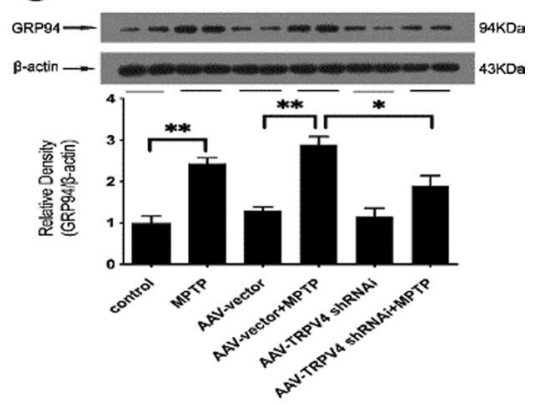

Fig. 5 TRPV4 knockdown attenuated MPTP-induced ER stress in the SN. After infused with 0.5 $\mu$ I AAV-TRPV4 shRNAi bilaterally into the SN of mice for 21 days and administrated with MPTP at 30 mg/kg/day for 7 days, the SN was rapidly dissected out. The protein levels of SERCA2, GRP78, GRP94, CHOP and procaspase-12 in the SN of mice were detected by Western blot. A Knockdown of TRPV4 restored the MPTP-induced decrease in SERCA2 $(n=6)$. B Knockdown of TRPV4 inhibited the MPTP-induced upregulation of GRP78 $(n=6)$. C Knockdown of TRPV4 inhibited the MPTP-induced upregulation of GRP94 ( $n=6$ ). D Knockdown of TRPV4 inhibited the MPTP-induced upregulation of CHOP ( $n=6)$. E Knockdown of TRPV4 partly inhibited the MPTP-induced decrease in procaspase-12 $(n=6)$. All data are presented as mean \pm SEM. Statistical significance: ${ }^{*} P<0.05,{ }^{* *} P<0.01$, ${ }^{* * *} P<0.001$

TRPV4 may mediate the inflammatory pathways and knockdown of TRPV4 has an anti-inflammatory effect.

\section{Upregulation of TRPV4 in the SN accelerated inflammation induced by MPTP}

To further confirm the role of TRPV4 in mediating the inflammatory pathways in the SN, we upregulated the expression of TRPV4 via AAV2/9 TRPV4 injection into the SN. Indeed, upregulation of TRPV4 induced inflammation. Activated TRPV4 significantly induced a reduction in procaspase-1 (Fig. 8A) and increased the levels of IL-18, COX-2, and 5-LOX (Fig. 8B-D). Moreover, we found that upregulation of TRPV4 in the SN exacerbated inflammation in PD mice. These data indicate that TRPV4 is an independent mediator of inflammation in the $\mathrm{SN}$ and its effect does not rely on MPTP-induced neurotoxicity.

\section{Discussion}

TRPV4 channels are novel plasma membrane $\mathrm{Ca}^{2+}$ channels that are widely expressed in the brain [34]. In the current study, we first found that in the SN of MPTP-induced PD mice, the expression of TRPV4 was increased. As the normal function of $\mathrm{Ca}^{2+}$ channels is essential for neuronal cells, disruption of these channels might lead to several diseases, including PD [35]. To directly evaluate the contribution of TRPV4 to neurogenic damage, we used the AAV injection to mediate TRPV4 expression in the SN (Fig. 1). We found that genetic knockdown of TRPV4 alleviated locomotor deficits in MPTP-treated mice, while upregulation of TRPV4 exacerbated MPTP-induced locomotor deficits (Fig. 2). To investigate whether TRPV4 plays a role in the pathogenesis of PD mice, immunohistochemistry was performed to assess the changes in the number of $\mathrm{TH}$-positive neurons in each group. TH-positive neurons are considered functional DA neurons in the SN. Mice treated with MPTP showed a decrease in the 

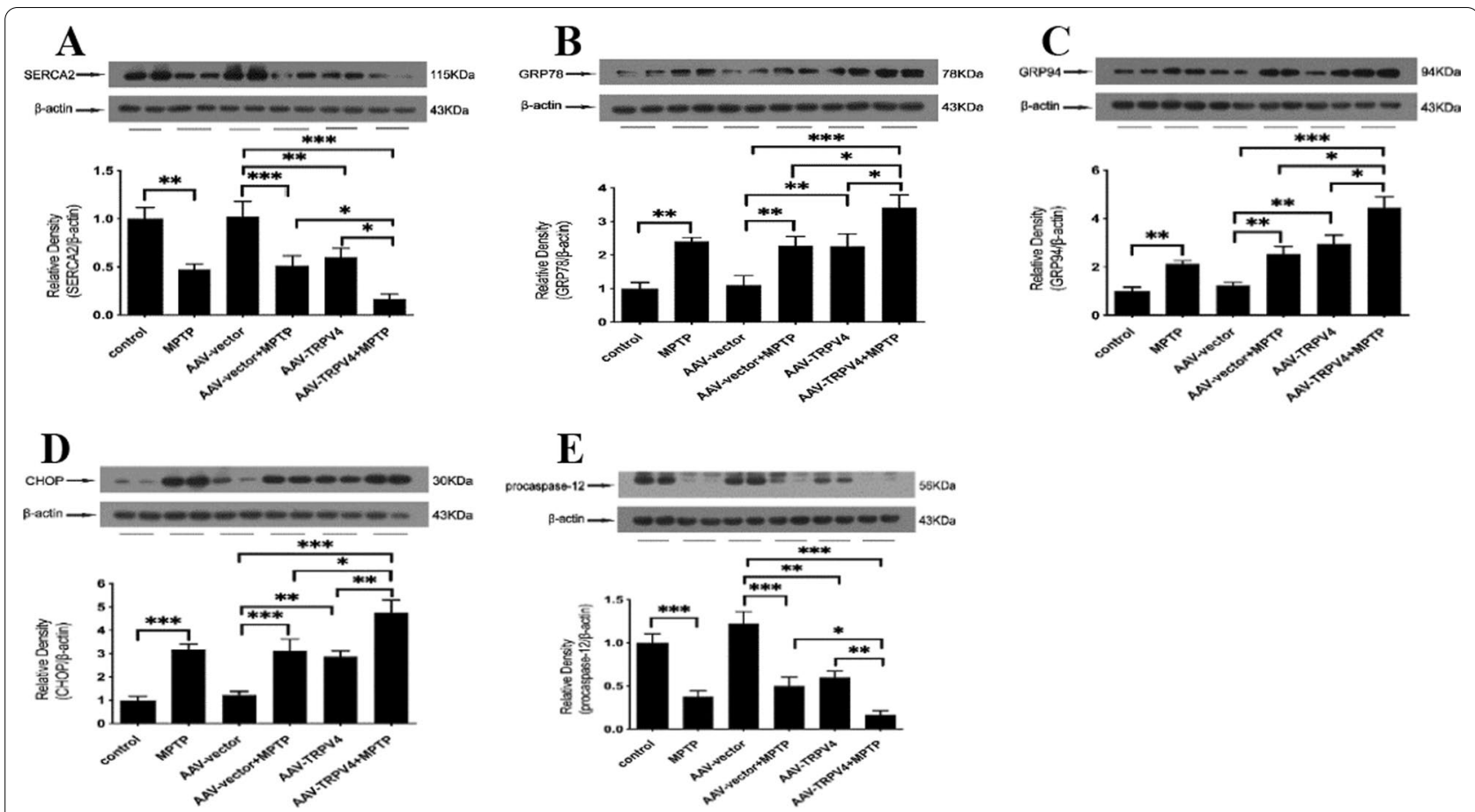

Fig. 6 Effects of TRPV4 upregulation on MPTP-induced ER stress in the SN. After infused with $0.3 \mu$ AAV-TRPV4 bilaterally into the SN of mice for 21 days and administrated with MPTP at $30 \mathrm{mg} / \mathrm{kg} /$ day for 7 days, the SN was rapidly dissected out. The protein levels of SERCA2, GRP78, GRP94, CHOP and procaspase-12 in the SN of mice were detected by Western blot. A Upregulation of TRPV4 deteriorated the MPTP-induced reduction in SERCA2 in the SN ( $n=6)$. B Upregulation of TRPV4 exacerbated the MPTP-induced activation of GRP78 in the SN $(n=6)$. C Upregulation of TRPV4 exacerbated the MPTP-induced activation of GRP94 in the SN $(n=6)$. D Upregulation of TRPV4 exacerbated the MPTP-induced activation of CHOP in the SN $(n=6)$. E Upregulation of TRPV4 accelerated the MPTP-induced decrease in procaspase-12 in the SN $(n=6)$. All data are presented as mean \pm SEM. Statistical significance: ${ }^{*} P<0.05,{ }^{* *} P<0.01,{ }^{* * *} P<0.001$

number of TH-positive neurons and Nissl-positive neurons. This phenomenon, which is the characteristic pathology of neurodegeneration in PD, is usually seen in the PD brain. Interestingly, knockdown of TRPV4 significantly increased TH-positive neuron and Nisslpositive neuron survival. Moreover, upregulation of TRPV4 further decreased the number of TH-positive neurons and Nissl-positive neurons in the $\mathrm{SN}$ of the PD brain (Figs. 3, 4). Altogether, these results confirmed that TRPV4 was responsible for the MPTP-induced PD phenotype.

To understand the neurotoxic role of TRPV4 in the MPTP-induced mouse model of PD, we investigated the mechanisms necessary for neuronal death. According to our previous studies in the $\mathrm{MPP}^{+}$-induced $\mathrm{PC} 12$ cell model in vitro, we measured the expression of molecules related to the ER-associated signaling pathway, which is a landmark of PD pathogenesis. Similar to ryanodine receptor (RyR) and inositol $(1,4,5)$-trisphosphate receptor $\left(\mathrm{IP}_{3} \mathrm{R}\right)$, which are $\mathrm{Ca}^{2+}$ release channels located in the ER, SERCA is a $\mathrm{Ca}^{2+}$ pump in the ER. It functions as a gatekeeper to bring $\mathrm{Ca}^{2+}$ into the ER to prevent $\mathrm{Ca}^{2+}$ homeostasis dysfunction. The major subtype SERCA2 is constitutively expressed in the brain [36]. It has been reported that TRPV4 expression upregulation by infrared radiation can trigger $\mathrm{Ca}^{2+}$-induced $\mathrm{Ca}^{2+}$ release (CICR) through the ryanodine receptor (RyR) and inositol (1,4,5)-trisphosphate receptor $\left(\mathrm{IP}_{3} \mathrm{R}\right)$ in neurons [37]. In the present study, we found that MPTP impaired $\mathrm{Ca}^{2+}$ buffering capacity by reducing SERCA2 expression, which then led to ER stress. Knockdown of TRPV4 by AAV significantly improved the function of SERCA2 and offset the cytoplasmic $\mathrm{Ca}^{2+}$ disturbance. The possible mechanism is that SERCA2 expression is destroyed by MPTPinduced inflammation and oxidative stress [38, 39], while TRPV4 inhibition has been identified to alleviate inflammation and oxidative stress $[25,40]$. In addition, GRP78 and GRP94 are key molecular chaperones of ER stress that play crucial roles in apoptosis of DA 

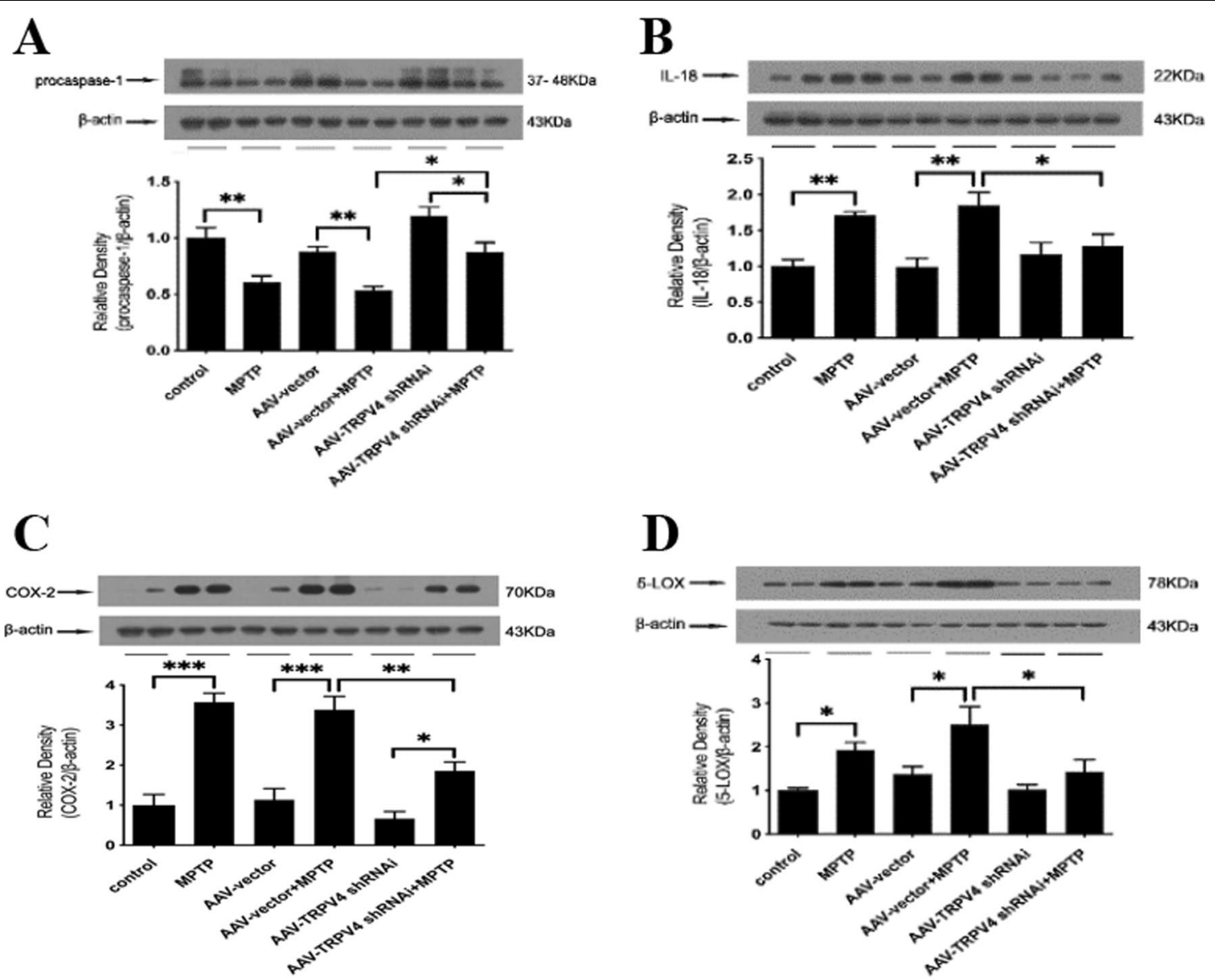

Fig. 7 Knockdown of TRPV4 in the SN attenuated inflammation in PD mice. After infused with $0.5 \mu$ AAV-TRPV4 shRNAi bilaterally into the SN of mice for 21 days and administrated with MPTP at $30 \mathrm{mg} / \mathrm{kg} /$ day for 7 days, the SN was rapidly dissected out. The protein levels of procaspase-1, IL-18, COX-2 and 5-LOX in the SN of mice were detected by Western blot. A Knockdown of TRPV4 in the SN restored the MPTP-induced the reduction in procaspase-1 $(n=6)$. B Knockdown of TRPV4 in the SN decreased the MPTP-induced high level of IL-18 $(n=6)$. C Knockdown of TRPV4 in the SN decreased the MPTP-induced high level of COX-2 $(n=6)$. D Knockdown of TRPV4 in the SN decreased the MPTP-induced high level of 5-LOX $(n=6)$. All data are presented as mean \pm SEM. Statistical significance: ${ }^{*} P<0.05,{ }^{* *} P<0.01,{ }^{* * *} P<0.001$

neurons $[41,42]$. It is well established that the protein levels of GRP78 and GRP94 are significantly decreased, whereas SERCA is upregulated in the dysfunctional hearts of mice [43]. This suggests that SERCA2 may be upstream of GRP78 and GRP94. In present study, GRP78 and GRP94 were markedly increased in the MPTP-treated group compared with the control group, which suggested that ER stress was induced by MPTP. Mediating TRPV4 expression in the SN by AAV significantly altered the levels of SERCA2, GRP78 and GRP94 (Figs. 5A-C and 6A-C). These results indicate that TRPV4 can mediate ER stress. To confirm our conclusions, we measured the levels of ER stress-associated apoptosis marker proteins, such as $\mathrm{CHOP}$ and procaspase-12 [44, 45]. Interestingly, we found that excessive
ER stress was reversibly suppressed by knockdown of TRPV4 through decreasing CHOP expression and procaspase-12 activation (Fig. 5D, E). However, upregulation of TRPV4 led to the opposite outcome (Fig. 6D, E). In summary, we found that TRPV4-mediated ER stress was at least one of the mechanisms of MPTP-induced neuronal injury.

The development of effective treatments for PD partly depends on a better understanding of the regulatory mechanisms. Moreover, numerous findings from clinical studies have indicated that inflammation is one of the pathogenic mechanisms of the development of PD. It is well established that MPTP causes inflammatory responses in vivo $[46,47]$. Several studies have shown the important role of TRPV4 in inflammation 

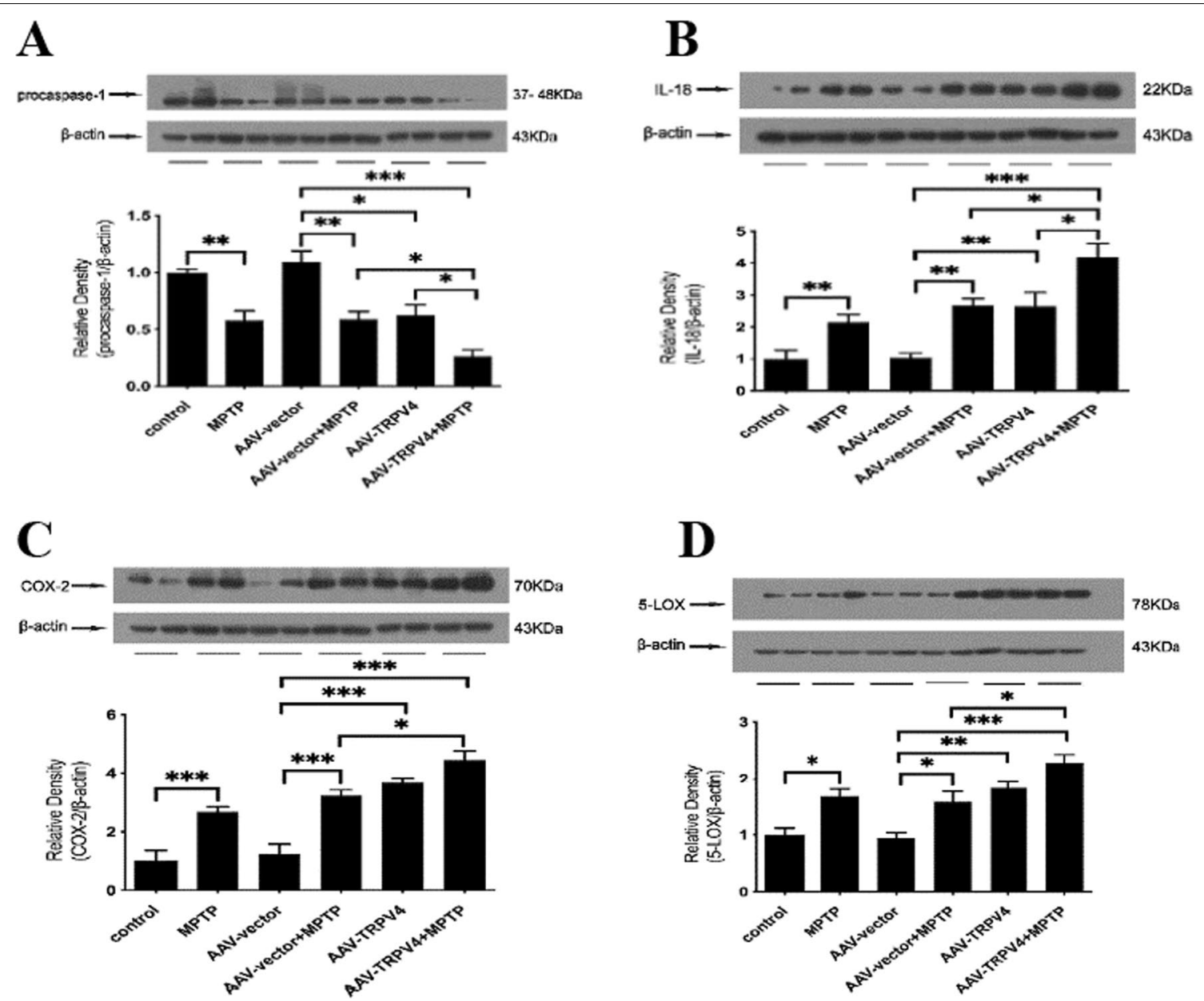

Fig. 8 Upregulation of TRPV4 in the SN aggravated inflammation in PD mice. After infused with $0.3 \mu \mathrm{I}$ AAV-TRPV4 bilaterally into the SN of mice for 21 days and administrated with MPTP at $30 \mathrm{mg} / \mathrm{kg} /$ day for 7 days, the SN was rapidly dissected out. The protein levels of procaspase-1, IL-18, COX-2 and 5-LOX in the SN of mice were detected by Western blot. A Upregulation of TRPV4 further decreased the MPTP-induced the reduction in procaspase-1 in the SN $(n=6)$. B Upregulation of TRPV4 further increased the MPTP-induced high level of IL-18 in the SN $(n=6)$. C Upregulation of TRPV4 further increased the MPTP-induced high level of COX- 2 in the SN $(n=6)$. D Upregulation of TRPV4 further increased the MPTP-induced high level of 5-LOX in the SN $(n=6)$. All data are presented as mean \pm SEM. Statistical significance: ${ }^{*} P<0.05,{ }^{* *} P<0.01$, ${ }^{* * *} P<0.001$

in different organs. It is considered a proinflammatory molecule under certain physiological conditions [4749]. The maturation of IL-18 and caspase- 1 depends on the activation of the nucleotide-binding oligomerization domain-like receptor family pyrin domain-containing 3 (NLRP3) inflammasome. Some factors, such as the elevation of intracellular free calcium concentration and lysosome disruption, are responsible for the activation of this kind of inflammasome [50]. A study proved that the TRPV4 specific inhibitor HC067047 could block the increases in NLRP3 and caspase-1 following pilocarpine-induced status epilepticus in mice, and the experimental results are consistent with our data [25]. Normally, endogenous activators of TRPV4 include heat, an acidic $\mathrm{pH}$, oxidative stress and arachidonic acid (AA) [19]. AA is released by cytoplasmic phospholipase $\mathrm{A}_{2}\left(\mathrm{PLA}_{2}\right)$ and then converted into inflammatory eicosanoids by cyclooxygenase (COX) and lipoxygenase (LOX). Various studies indicate that TRPV4 signaling requires activation of $\mathrm{PLA}_{2}[51,52]$, and $\mathrm{Ca}^{2+}$-dependent protein kinases activated by the influx of $\mathrm{Ca}^{2+}\left(\left[\mathrm{Ca}^{2+}\right]_{\mathrm{i}}\right)$ may induce COX and LOX overexpression [53-55]. Subsequently, upregulation of COX and LOX drives the production of proinflammatory factors, including prostaglandin (PG) [52] and leukotriene (LT) [56], which are linked to inflammation and inflammatory injury. This evidence demonstrates that TRPV4-depended $\left[\mathrm{Ca}^{2+}\right]_{\mathrm{i}}$ plays a crucial role in the 
AA metabolic process, which causes an inflammatory storm. In present study, we first investigated the role of TRPV4 in inflammation in the SN of PD mice. To confirm our hypothesis in this research, we measured the levels of proinflammatory molecules in the SN. We found that knockdown of TRPV4 significantly inhibited MPTP-induced activation of COX-2, 5-LOX, IL-18 and procaspase-1 (Fig. 7). In contrast, upregulation of TRPV4 significantly increased the levels of COX-2, 5-LOX, and IL-18 and decreased procaspase-1expression, which were deteriorated in PD mice (Fig. 8). These data support our hypothesis that TRPV4 acts as a mediator of neuroinflammation and may amplify the inflammatory responses.

$\mathrm{Ca}^{2+}$ is an important messenger responsible for cellular activities, and $\mathrm{Ca}^{2+}$ homeostasis dysfunction is commonly observed in neurological diseases. In present study, we found that TRPV4, a $\mathrm{Ca}^{2+}$-permeable channel, was involved in the MPTP-induced PD mouse model. Specifically, TRPV4 expression was upregulated in the SN by treatment with MPTP for the following reasons. First, TRPV4 was directly activated by $\mathrm{PLA}_{2}$ [57], the expression of which was upregulated by MPTP [58]. Second, TRPV4 was activated by mechanical stretching of the cell membrane and changes in extracellular osmolarity [11], which were elicited by MPTPinduced inflammatory edema.

In present study, we found that activated TRPV4 contributed to neuronal loss in the SN of PD mice via ER stress and the inflammatory pathway (Fig. 9). Notably,
ER stress and neuroinflammation interact in nervous system diseases $[59,60]$. It is well accepted that inflammation is activated by ER stress, and high expression of multiple proinflammatory factors is relevant to neurodegenerative diseases. Moreover, inflammation-induced ER stress is also involved in neurotoxicity. It has been proven that inhibiting ER stress attenuates inflammation-mediated neuronal dysfunction, and this effect ultimately decreases the expression of apoptosis-associated proteins in the inflammatory microenvironment [61]. Taken together, these results suggest that ER stress and inflammation are highly orchestrated processes. In present study, we found for the first time that (I) TRPV4 expression was upregulated in MPTP-induced mice. (II) TRPV4 mediated ER stress and inflammation to induce the loss of DA neurons and behavioral deficits in MPTPinduced mice, and knockdown of TRPV4 prevented these neuronal impairments. Therefore, this study may open new research avenues for the drug treatment of PD. However, the clinical application value and the potential of special chemical antagonists of TRPV4 or natural medicines and gene therapies targeting TRPV4 need to be investigated in the future.

\section{Conclusions}

The present study demonstrated that TRPV4 regulated ER stress and inflammation to induce the loss of DA neurons and behavioral deficits of MPTP-induced mice. Knockdown of TRPV4 alleviated these neuronal impairments significantly. Therefore, targeting TRPV4 to

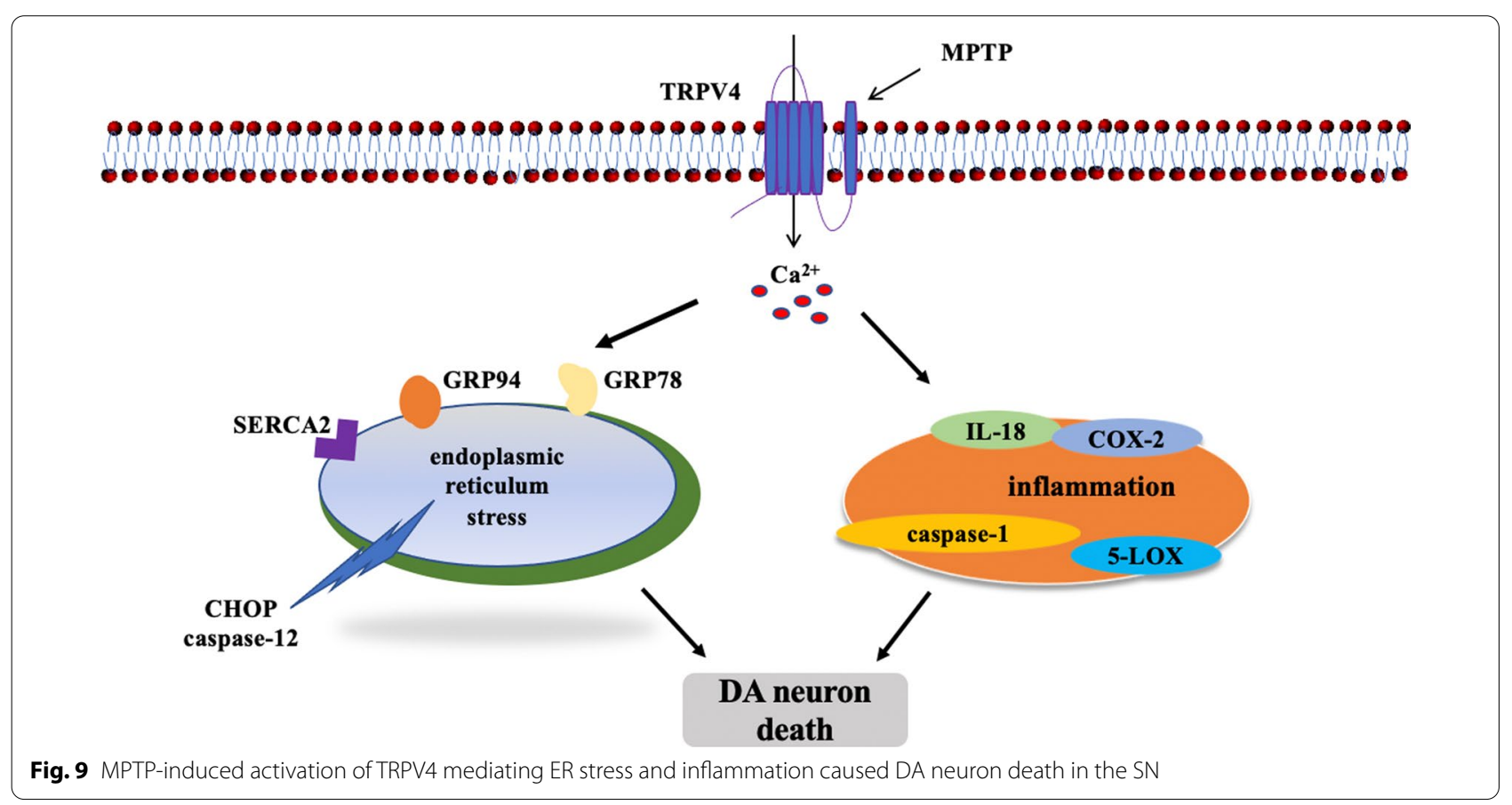




\section{mediate ER stress and inflammation may be a potential therapeutic strategy for PD.}

\begin{abstract}
Abbreviations
PD: Parkinson's disease; TRPV4: Transient receptor potential vanilloid 4; ER: Endoplasmic reticulum; MPTP: 1-Methyl-4-phenyl-1,2,3,6-tetrahydropyridine; AAV: Adeno-associated virus; SN: Substantia nigra; TH: Tyrosine hydroxylase; DA: Dopamine; MPP+: 1-Methyl-4-phenylpyridinium ion; IL-6: Interleukin-6; IL-1 $\beta$ : Interleukin-1 $\beta$; TNF-a: Tumor necrosis factor-a; TRP: Transient receptor potential; UPR: Unfolded protein response; SERCA2: Sarco/endoplasmic reticulum $\mathrm{Ca}^{2+}$-ATPase 2; GRP78: Glucose-regulated protein 78; GRP94: Glucose-regulated protein 94; procaspase-12: Pro-cysteinyl aspartate specific proteinase-12; CHOP: C/EBP homologous protein; procaspase-1: Pro-cysteinyl aspartate specific proteinase-1; COX-2: Cyclooxgenase-2; 5-LOX: 5-Lipoxygenase; IL-18: Interleukin-18; CICR: $\mathrm{Ca}^{2+}{ }_{-}$induced $\mathrm{Ca}^{2+}$ release; RyR: Ryanodine receptor; IP $_{3}$ : Inositol (1,4,5)-trisphosphate receptor; SERCA: Sarco/endoplasmic reticulum $\mathrm{Ca}^{2+}$-ATPase; caspase-12: Cysteinyl aspartate specific proteinase-12; NLRP3: Nucleotide-binding oligomerization domain-like receptor family pyrin domain-containing 3; AA: Arachidonic acid; PLA 2 : Phospholipase $\mathrm{A}_{2}$; COX: Cyclooxgenase; LOX: Lipoxygenase.
\end{abstract}

\section{Acknowledgements}

We would like to thank Medical school of Kunming University of Science and Technology.

\section{Author contributions}

$\mathrm{NL}$ contributed to the conception and design of the study, researched and acquired the data, drafted the manuscript. LB, ZL, RG analyzed the data and drafted the figures. FY, DZ did the behavioral tests. JB organized this study and revised the manuscript. All authors read and approved the final manuscript.

\section{Funding}

This work was supported by National Natural Science Foundation of China (No. U2002220), the Yunling Scholar (No.1097821401) and the Oxidative Stress and Defense Key Laboratory in University of Yunnan Province (2018).

\section{Availability of data and materials}

All data generated or analyzed during this study are included in this published article.

\section{Declarations}

\section{Ethics approval}

All animal experiments were approved by the Committee on Animal Use and Protection of Yunnan province (No. LA2008305). The work was licensed under the animal ethics council of Kunming University of Science and Technology. All efforts were made to minimize pain and numbers of the animals used in these experiments.

\section{Consent for publication}

All authors agree to the publication of this manuscript.

\section{Competing interests}

All the authors declared no competing financial interests.

\section{Author details}

${ }^{1}$ Medical School, Kunming University of Science and Technology, No.727 Jingming South Road, Kunming 650500, China. ${ }^{2}$ Department of Anesthesiology, The First People's Hospital of Yunnan Province, The Affiliated Hospital of Kunming University of Science and Technology, Kunming 650032, China.

Received: 22 April 2021 Accepted: 10 January 2022

Published online: 29 January 2022

\section{References}

1. Lai F, Jiang R, Xie W, et al. Intestinal pathology and gut microbiota alterations in a methyl-4-phenyl-1,2,3,6-tetrahydropyridine (MPTP) mouse model of Parkinson's disease. Neurochem Res. 2018;43(10):1986-99.

2. Pupyshev AB, Tikhonova MA, Akopyan AA, et al. Therapeutic activation of autophagy by combined treatment with rapamycin and trehalose in a mouse MPTP-induced model of Parkinson's disease. Pharmacol Biochem Behav. 2019;177:1-11.

3. Zhu J, Dou S, Jiang Y, Chen J, et al. Apelin-13 protects dopaminergic neurons in MPTP-induced Parkinson's disease model mice through inhibiting endoplasmic reticulum stress and promoting autophagy. Brain Res. 2019;1715:203-12.

4. Li Y, Liu Z, Wang D, et al. Ucf-101 protects in vivoandin vitro models of PD against 6-hydroxydopamine toxicity by alleviating endoplasmic reticulum stress via the Wnt/ $\beta$-catenin pathway. J Clin Neurosci. 2020;71:217-25.

5. Liu N, Liu J, Wen X, et al. TRPV4 contributes to ER stress: relation to apoptosis in the $\mathrm{MPP}^{+}$-induced cell model of Parkinson's disease. Life Sci. 2020;261:118461.

6. Dufek M, Hamanová M, Lokaj J, et al. Serum inflammatory biomarkers in Parkinson's disease. Parkinsonism Relat Disord. 2009:15(4):318-20.

7. Chen X, Hu Y, Cao Z, et al. Cerebrospinal fluid inflammatory cytokine aberrations in Alzheimer's disease, Parkinson's disease and amyotrophic lateral sclerosis: a systematic review and meta-analysis. Front Immunol. 2018:9:2122.

8. Corrigan F, Mander KA, Leonard AV, et al. Neurogenic inflammation after traumatic brain injury and its potentiation of classical inflammation. J Neuroinflamm. 2016:13(1):264.

9. Das A, Wallace GC, Holmes C, et al. Hippocampal tissue of patients with refractory temporal lobe epilepsy is associated with astrocyte activation, inflammation, and altered expression of channels and receptors. Neuroscience. 2012;220:237-46.

10. Osmanlıoğlu H, Yıldırım MK, Akyuva Y, et al. Morphine induces apoptosis, inflammation, and mitochondrial oxidative stress via activation of TRPM2 channel and nitric oxide signaling pathways in the hippocampus. Mol Neurobiol. 2020;57(8):3376-89.

11. Garcia-Elias A, Mrkonjić S, Jung C, et al. The TRPV4 channel. Handb Exp Pharmacol. 2014;222:293-319.

12. Shibasaki K, Yamada K, Miwa H, et al. Temperature elevation in epileptogenic foci exacerbates epileptic discharge through TRPV4 activation. Lab Invest. 2020;100(2):274-84.

13. Sugawara S, Shinoda M, Hayashi Y, et al. Increase in IGF-1 expression in the injured infraorbital nerve and possible implications for orofacial neuropathic pain. Int J Mol Sci. 2019;20(24):6360.

14. Baratchi S, Keov P, Darby WG, et al. The TRPV4 agonist GSK1016790A regulates the membrane expression of TRPV4 channels. Front Pharmacol. 2019:10:6.

15. Veteto $A B$, Peana $D$, Lambert MD, et al. TRPV4 contributes to stretchinduced hypercontractility and time-dependent dysfunction in the aged heart. Cardiovasc Res. 2020;116(11):1887-96.

16. Dong $Q, L i J, W u Q F$, et al. Blockage of transient receptor potential vanilloid 4 alleviates myocardial ischemia/reperfusion injury in mice. Sci Rep. 2017;7:42678.

17. Olivan-Viguera A, Garcia-Otin AL, Lozano-Gerona J, et al. Pharmacological activation of TRPV 4 produces immediate cell damage and induction of apoptosis in human melanoma cells and HaCaT keratinocytes. PLoS ONE. 2018;13(1):e0190307.

18. Liu M, Liu X, Wang L, et al. TRPV4 inhibition improved myelination and reduced glia reactivity and inflammation in a cuprizone-induced mouse model of demyelination. Front Cell Neurosci. 2018;12:392.

19. Liu N, Wu J, Chen Y, et al. Channels that cooperate with TRPV4 in the brain. J Mol Neurosci. 2020;70(11):1812-20.

20. Jie P, Hong Z, Tian Y, et al. Activation of transient receptor potential vanilloid 4 induces apoptosis in hippocampus through downregulating PI3K/Akt and upregulating p38 MAPK signaling pathways. Cell Death Dis. 2015;6(6):e1775.

21. Jie $\mathrm{P}, \mathrm{Lu} Z$, Hong Z, et al. Activation of transient receptor potential vanilloid 4 is involved in neuronal injury in middle cerebral artery occlusion in mice. Mol Neurobiol. 2016:53(1):8-17. 
22. Shen J, Tu L, Chen D, et al. TRPV4 channels stimulate $\mathrm{Ca}^{2+}$-induced $\mathrm{Ca}^{2+}$ release in mouse neurons and trigger endoplasmic reticulum stress after intracerebral hemorrhage. Brain Res Bull. 2019;146:143-52.

23. Jie $\mathrm{P}$, Tian $\mathrm{Y}$, Hong $Z$, et al. Blockage of transient receptor potential vanilloid 4 inhibits brain edema in middle cerebral artery occlusion mice. Front Cell Neurosci. 2015;9:141.

24. Lu KT, Huang TC, Tsai YH, et al. Transient receptor potential vanilloid type 4 channels mediate $\mathrm{Na}-\mathrm{K}-\mathrm{Cl}$-co-transporter-induced brain edema after traumatic brain injury. J Neurochem. 2017;140(5):718-27.

25. Wang Z, Zhou L, An D, et al. TRPV4-induced inflammatory response is involved in neuronal death in pilocarpine model of temporal lobe epilepsy in mice. Cell Death Dis. 2019;10(6):386.

26. Lee JC, Choe SY. Age-related changes in the distribution of transient receptor potential vanilloid 4 channel (TRPV4) in the central nervous system of rats. J Mol Histol. 2014;45(5):497-505.

27. Bai JZ, Lipski J. Involvement of TRPV4 channels in A $(40)$-induced hippocampal cell death and astrocytic $\mathrm{Ca}(2+)$ signalling. Neurotoxicology. 2014;41:64-72.

28. Espay AJ, Morgante F, Merola A, et al. Levodopa-induced dyskinesia in Parkinson disease: current and evolving concepts. Ann Neurol. 2018;84(6):797-811.

29. Salamon A, Zádori D, Szpisjak L, et al. Opicapone for the treatment of Parkinson's disease: an update. Expert Opin Pharmacother. 2019;20(18):2201-7.

30. Su Y, Deng MF, Xiong W, et al. MicroRNA-26a/death-associated protein kinase 1 signaling induces synucleinopathy and dopaminergic neuron degeneration in Parkinson's disease. Biol Psychiatry. 2019;85(9):769-81.

31. Zhang X, Bai L, Zhang S, et al. TrX-1 ameliorates learning and memory deficits in MPTP-induced Parkinson's disease model in mice. Free Radic Biol Med. 2018;124:380-7.

32. Sgadò P, Viaggi C, Pinna A, et al. Behavioral, neurochemical, and electrophysiological changes in an early spontaneous mouse model of nigrostriatal degeneration. Neurotox Res. 2011;20(2):170-81.

33. Meredith GE, Kang UJ. Behavioral models of Parkinson's disease in rodents: a new look at an old problem. Mov Disord. 2006;21(10):1595-606.

34. Liu N, Yan F, Ma Q, et al. Modulation of TRPV4 and BKCa for treatment of brain diseases. Bioorg Med Chem. 2020;28(16):115609.

35. Surmeier DJ, Schumacker PT, Guzman JD, et al. Calcium and Parkinson's disease. Biochem Biophys Res Commun. 2017;483(4):1013-9.

36. Britzolaki A, Saurine J, Flaherty E, et al. The SERCA2: a gatekeeper of neuronal calcium homeostasis in the brain. Cell Mol Neurobiol. 2018;38(5):981-94.

37. Barrett JN, Rincon S, Singh J, et al. Pulsed infrared releases $\mathrm{Ca}^{2+}$ from the endoplasmic reticulum of cultured spiral ganglion neurons. J Neurophysiol. 2018;120(2):509-24

38. Bigelow DJ. Nitrotyrosine-modified SERCA2: a cellular sensor of reactive nitrogen species. Pflugers Arch. 2009;457(3):701-10.

39. Balderas-Villalobos J, Molina-Muñoz T, Mailloux-Salinas P, et al. Oxidative stress in cardiomyocytes contributes to decreased SERCA2a activity in rats with metabolic syndrome. Am J Physiol Heart Circ Physiol. 2013;305(9):H1344-53.

40. Hong Z, Tian Y, Yuan Y, et al. Enhanced oxidative stress is responsible for TRPV4-induced neurotoxicity. Front Cell Neurosci. 2016;10:232.

41. Rana SVS. Endoplasmic reticulum stress induced by toxic elements-a review of recent developments. Biol Trace Elem Res. 2020;196(1):10-9.

42. Zeng XS, Jia JJ, Kwon Y, et al. The role of thioredoxin-1 in suppression of endoplasmic reticulum stress in Parkinson disease. Free Radic Biol Med. 2014;67:10-8

43. Zhang $L$, Yan $X$, Zhang $Y L$, et al. Vitamin $D$ attenuates pressure overloadinduced cardiac remodeling and dysfunction in mice. J Steroid Biochem Mol Biol. 2018;178:293-302.

44. Modi J, Menzie-Suderam J, Xu H, et al. Mode of action of granulocytecolony stimulating factor (G-CSF) as a novel therapy for stroke in a mouse model. J Biomed Sci. 2020;27(1):19.

45. Kamarehei M, Kabudanian Ardestani S, Firouzi M, et al. Increased expression of endoplasmic reticulum stress-related caspase-12 and CHOP in the hippocampus of EAE mice. Brain Res Bull. 2019;147:174-82.

46. Xie W, Gao J, Jiang R, et al. Twice subacute MPTP administrations induced time-dependent dopaminergic neurodegeneration and inflammation in midbrain and ileum, as well as gut microbiota disorders in PD mice. Neurotoxicology. 2020;76:200-12.

47. Javed $H$, Thangavel $R$, Selvakumar GP, et al. NLRP3 inflammasome and glia maturation factor coordinately regulate neuroinflammation and neuronal loss in MPTP mouse model of Parkinson's disease. Int Immunopharmacol. 2020;83:106441.

48. Scheraga RG, Southern BD, Grove LM, et al. The role of TRPV4 in regulating innate immune cell function in lung inflammation. Front Immunol. 2020;11:1211.

49. Vergnolle N. TRPV4: new therapeutic target for inflammatory bowel diseases. Biochem Pharmacol. 2014;89(2):157-61.

50. Jin C, Flavell RA. Molecular mechanism of NLRP3 inflammasome activation. J Clin Immunol. 2010;30(5):628-31.

51. Redmon SN, Yarishkin O, Lakk M, et al. TRPV4 channels mediate the mechanoresponse in retinal microglia. Glia. 2021;69(6):1563-82.

52. Marrelli SP, O'neil RG, Brown RC, et al. PLA2 and TRPV4 channels regulate endothelial calcium in cerebral arteries. Am J Physiol Heart Circ Physiol. 2007:292(3):H1390-7.

53. Lin CC, Hsieh HL, Chi PL, et al. Upregulation of COX-2/PGE2 by ET-1 mediated through $\mathrm{Ca}^{2+}$-dependent signals in mouse brain microvascular endothelial cells. Mol Neurobiol. 2014;49(3):1256-69.

54. Kim JA, Chung YJ, Lee YS. Intracellular $\mathrm{Ca}^{2+}$ mediates lipoxygenaseinduced proliferation of U-373 MG human astrocytoma cells. Arch Pharm Res. 1998;21(6):664-70.

55. Kang KH, Liou HH, Hour MJ, et al. Protection of dopaminergic neurons by 5-lipoxygenase inhibitor. Neuropharmacology. 2013;73:380-7.

56. Romp E, Arakandy V, Fischer J, et al. Exotoxins from Staphylococcus aureus activate 5-lipoxygenase and induce leukotriene biosynthesis. Cell Mol Life Sci. 2020;77(19):3841-58.

57. Vriens J, Watanabe $H$, Janssens $A$, et al. Cell swelling, heat, and chemical agonists use distinct pathways for the activation of the cation channel TRPV4. Proc Natl Acad Sci USA. 2004;101(1):396-401.

58. Tariq M, Khan HA, Al Moutaery $K$, et al. Protective effect of quinacrine on striatal dopamine levels in 6-OHDA and MPTP models of Parkinsonism in rodents. Brain Res Bull. 2001;54(1):77-82.

59. Yang $L$, Guo $Y$, Huang $M$, et al. Thioredoxin-1 protects spinal cord from demyelination induced by methamphetamine through suppressing endoplasmic reticulum stress and inflammation. Front Neurol. 2018:9:49.

60. Meares GP, Liu Y, Rajbhandari R, et al. PERK-dependent activation of JAK1 and STAT3 contributes to endoplasmic reticulum stress-induced inflammation. Mol Cell Biol. 2014;34(20):3911-25.

61. Hou S, Wang L, Zhang G. Mitofusin-2 regulates inflammation-mediated mouse neuroblastoma N2a cells dysfunction and endoplasmic reticulum stress via the Yap-Hippo pathway. J Physiol Sci. 2019;69(5):697-709.

\section{Publisher's Note}

Springer Nature remains neutral with regard to jurisdictional claims in published maps and institutional affiliations.

Ready to submit your research? Choose BMC and benefit from:

- fast, convenient online submission

- thorough peer review by experienced researchers in your field

- rapid publication on acceptance

- support for research data, including large and complex data types

- gold Open Access which fosters wider collaboration and increased citations

- maximum visibility for your research: over $100 \mathrm{M}$ website views per year

At BMC, research is always in progress.

Learn more biomedcentral.com/submissions 